\title{
Turquie : des intentions aux réalités
}

Joëlle Schwendimann Cassel et Eser Gerdanli

\section{OpenEdition}

Journals

Édition électronique

URL : http://journals.openedition.org/ries/3978

DOI : $10.4000 /$ ries.3978

ISSN : 2261-4265

\section{Éditeur}

Centre international d'études pédagogiques

\section{Édition imprimée}

Date de publication : 7 septembre 1995

Pagination : 131-136

ISSN : 1254-4590

\section{Référence électronique}

Joëlle Schwendimann Cassel et Eser Gerdanli, «Turquie : des intentions aux réalités », Revue

internationale d'éducation de Sèvres [En ligne], 07 | 1995, mis en ligne le , consulté le 14 novembre 2019. URL : http://journals.openedition.org/ries/3978 ; DOI : 10.4000/ries.3978

Ce document a été généré automatiquement le 14 novembre 2019.

(c) Tous droits réservés 


\title{
Turquie: des intentions aux réalités
}

\author{
Joëlle Schwendimann Cassel et Eser Gerdanli
}

1 En Turquie, on dit : « Un homme qui connaît une langue étrangère en vaut deux » comme dit le proverbe français: "Un homme avisé en vaut deux»; et dans cet esprit, une deuxième langue est associée à l'image d'un pont qui permet de franchir les frontières, d'aller plus loin et d'enrichir ses connaissances.

2 Nous nous proposons de retracer en quelques lignes l'historique de l'enseignement bilingue en Turquie et de décrire la situation actuelle. Nous évoquerons en particulier le contexte éducatif des établissements francophones de Turquie.

\section{Historique}

3 Dans l'histoire de la Turquie, c'est avec la France que les premières relations avec les pays occidentaux ont été construites. Les intellectuels turcs de l'époque étaient fortement influencés par la littérature et la Révolution françaises.

4 Les premiers contacts éducatifs ont été établis pendant les travaux de réorganisation dans l'armée ottomane. Le ministre des affaires étrangères de l'époque, lors de sa visite officielle en France, avait rencontré des étudiants turcs dont la plupart venaient des écoles militaires, et avaient l'intention d'améliorer leur français avant d'être reçus dans les grandes écoles. D'autre part, les relations qui se développaient avec l'Occident après la déclaration du " décret impérial de Gülhané », prévoyant une grande réorganisation des institutions officielles et publiques, faisaient sentir le besoin d'un grand nombre de fonctionnaires et hauts fonctionnaires "polyglottes ». Il s'agissait d'une grande réforme qui prenait les institutions de l'Occident comme modèle.

5 L'ambassadeur de l'Empire ottoman à Paris a convaincu le ministre des affaires étrangères : ils ont fondé «l'École ottomane de Paris » et la fondation du célèbre lycée Galatasaray a suivi la déclaration du traité de Paris en 1856. 
Un grand nombre des diplômés de ce lycée ont continué leurs études en France, à l'École libre des sciences politiques qui a été ouverte quelques années plus tard, et ils ont formé l'intelligentsia de la jeune République turque.

7 L'enseignement bilingue qui a germé dans cette atmosphère a continué son chemin avec la fondation d'autres établissements francophones comme Saint-Michel, Saint-Benoît, Notre-Dame de Sion, Sainte-Pulchérie, Saint- Joseph, les lycées Tevfik Fikret d'Ankara et d'Izmir, tous privés à part le lycée Galatasaray, auxquels d'autres établissements qui enseignent l'anglais, l'allemand ou l'italien se sont rajoutés, pendant la première moitié du siècle. Mais le français a été la langue qui a donné son souffle à l'intellectualisme et à la littérature turque de l'époque largement empreinte de la littérature française.

\section{Intentions}

8 À l'heure actuelle, le but des parents qui planifient l'avenir de leurs enfants n'a pas autant changé : il s'agit de placer leurs enfants dans une école qui dispense un enseignement de qualité, qui prépare efficacement les élèves aux études supérieures et qui leur offre les clés d'un monde au-delà des frontières.

9 En effet, suivre une scolarité bilingue signifie pour l'enfant : s'initier progressivement à une langue et culture étrangères, acquérir des compétences d'expression et de compréhension dans cette langue mais aussi utiliser cette langue comme outil médiateur de savoirs et de savoir-faire.

L'habitude pour un élève de passer d'un système de symboles à un autre va stimuler le développement de ses fonctions cognitives et de ses capacités intellectuelles.

11 De plus, l'apport social et culturel de l'apprentissage dans et par une seconde langue n'est pas à négliger : chaque langue véhicule une idéologie, un système de valeurs, une certaine expression du monde et de la réalité, une façon d'être et de se comporter avec les autres, une façon de transmettre et de construire les savoirs. La faculté de gérer deux systèmes de langue, de pensée, de valeurs contribue à former des générations ouvertes au monde extérieur, sensibles aux civilisations mondiales et adaptées à l'internationalisation des relations commerciales et économiques.

12 Dans cette perspective, aujourd'hui, la connaissance d'au moins une langue étrangère est considérée comme un avantage tellement indispensable que le ministère de l'éducation s'est senti obligé d'appliquer sur le plan national un enseignement identique dans les écoles publiques et il a fondé les lycées dits « lycées anatoliens » dans plusieurs villes de l'Anatolie, avec un programme officiel qui prévoit l'étude renforcée d'une langue étrangère ; mais les établissements privés restent majoritaires parmi les établissements bilingues.

\section{Contexte éducatif}

13 Comme nous venons de le mentionner, deux types d'établissements assurent une scolarité bilingue. Les langues enseignées dans ces établissements sont l'anglais, l'allemand et le français. 


\section{Les établissements bilingues}

\section{Les lycées anatoliens}

14 Au sein du marché de l'emploi, une demande croissante pour les diplômés des lycées bilingues privés a poussé le ministère de l'éducation à prendre des mesures en ouvrant des lycées bilingues publics. Ces établissements que l'on appelle «lycées anatoliens» recrutent leurs élèves à partir d'un concours national à la fin de l'école primaire sous forme de QCM. Les candidats font leur choix de langue et d'établissement au moment où ils déposent leur candidature. Selon les résultats obtenus, les élèves se répartissent dans des collèges de plus ou moins bon niveau.

15 Les lycées anatoliens, ainsi que le lycée Galatasaray, établissements bilingues publics de prestige en Turquie, recrutent en priorité les meilleurs élèves au concours national. Les élèves qui n'ont pas été suffisamment bien classés ou qui ne se sont pas présentés à ce concours s'inscriront dans les lycées d'enseignement général qui proposent un apprentissage optionnel de la langue étrangère.

Les lycées anatoliens sont soumis aux mêmes règlements que les établissements privés concernant l'enseignement de la langue étrangère: même nombre d'heures d'enseignement, mêmes matières enseignées en langue étrangère, possibilité d'utilisation des mêmes manuels scolaires en langue étrangère.

\section{Les établissements bilingues privés}

17 Ils sont localisés dans les trois plus grandes villes de Turquie : Istanbul, Ankara et Izmir. Ils recrutent des enfants des classes socio-économiques favorisées, en raison du montant des frais de scolarité relativement élevé.

Les élèves sont sélectionnés à partir d'un concours d'entrée à l'issue de l'école primaire, différent du concours d'entrée des établissements bilingues publics (lycées anatoliens et Galatasaray).

19 Par contre, le système d'enseignement dans les deux types d'établissements - public/ privé - est identique.

20 La scolarité débute par une classe préparatoire appelée Hazirlik consacrée à l'apprentissage intensif de la langue étrangère à raison de vingt-quatre heures par semaine. La durée de ce cycle préparatoire varie suivant l'établissement entre une ou deux années.

21 Le nombre d'heures consacrées à l'enseignement de la deuxième langue diminue dans les classes du collège et du lycée, par contre les élèves suivent l'enseignement des mathématiques, des sciences physiques et de la biologie dans cette langue étrangère. Les autres matières continuent d'être enseignées en turc.

22 Le règlement intérieur ainsi que les programmes suivis sont conformes aux exigences $d u$ ministère de l'éducation nationale turque.

23 Depuis quelques années, on assiste à la mise en place de sections primaires au sein de ces établissements afin d'assurer un cycle complet d'enseignement bilingue; mais ces établissements gardent toujours leurs classes préparatoires pour les élèves qu'ils recrutent après l'école primaire. 


\section{Situation des langues étrangères}

Parmi les trois langues enseignées, le français, qui était en tête de liste jusque dans les années 1950, a été devancé par l'anglais et l'allemand dans les établissements bilingues.

Si la situation du français dans les établissements privés et au lycée Galatasaray reste globalement bonne, les lycées anatoliens francophones par contre connaissent une situation de plus en plus difficile, liée à une démotivation de la part des élèves. En effet, les représentations et les valeurs associées à la langue française souffrent de la concurrence de l'anglais et de l'allemand, langues plus prometteuses si on se place au niveau des échanges commerciaux et économiques.

Ainsi, l'anglais, qui occupe incontestablement la première place, n'est pas touché par les problèmes que connaît l'enseignement $d u$ français.

Quant à l'allemand, il connaît un redressement et un parcours croissants dans les réseaux public et privé : en effet, le nombre d'élèves est en hausse. Une des raisons principales, outre les raisons d'ordre économique est le retour au pays des enfants germanophones des immigrés turcs. Cette mesure de retour au pays est soutenue par le gouvernement allemand grâce à la mise à disposition d'un nombre important d'enseignants allemands.

\section{L'enseignement bilingue franco-turc}

La plupart des jeunes qui apprennent le français ne visent pas pour la plupart à continuer leurs études en France, car le coût en est trop élevé. Ils préfèrent faire leurs études universitaires dans leur pays. Mais il faut reconnaitre que les universités qui pour l'instant jouissent de la meilleure réputation sont anglophones.

Par ailleurs, pour pouvoir s'inscrire à l'université l'étudiant est obligé de passer un concours national très sélectif. C'est souvent au niveau des deux dernières années d'études secondaires que le lycéen, pour qui l'intérêt prédominant est de réussir le concours d'entrée à l'université, rompt le contact avec le français et consacre toute sa volonté d'apprentissage aux matières scientifiques, parce que la sélection des facultés les plus recherchées se fait dans ce domaine.

30 L'élève ne tarde pas à vivre le paradoxe entre l'idée de se préparer à un avenir plus solide mais plus difficile dont il pourra recueillir les fruits plus tard et l'idée de se garantir une bonne place à l'université en se contentant d'un français de niveau moyen.

De plus l'âge de l'adolescence correspond souvent à une période d'opposition aux valeurs parentales. Or, le choix de leur cursus bilingue relève pour la plupart d'une décision parentale. Il arrive alors que l'élève développe à l'encontre de la langue et de la culture étrangère des attitudes de rejet et de refus parce qu'elles sont alors vécues comme une menace par rapport à leur langue mais surtout à leur culture d'origine.

\section{Les moyens d'enseignement}

32 Le ministère des affaires étrangères français détache un certain nombre d'enseignants français auprès de ces établissements bilingues (publics et privés) chargés d'une partie de l'enseignement du français et en français. 
Les autres enseignants sont recrutés localement au niveau du pays, soit parmi des natifs bilingues, soit parmi des francophones compétents de passage.

Trouver des enseignants bilingues franco-turcs d'un niveau satisfaisant devient de plus en plus difficile, aussi bien dans le domaine des sciences que dans le domaine de la langue. Les sections de langue qui forment les enseignants de français à l'université proposent un enseignement plutôt livresque axé sur l'enseignement de la littérature alors que la plupart des étudiants, à cause d'un niveau de maîtrise moyen voire faible de la langue française, ne peuvent pas profiter pleinement de cet enseignement. Par ailleurs ces étudiants sont peu formés à la méthodologie du français langue étrangère. Ce manque de formation et de compétence entraîne la démotivation des élèves.

Les étudiants de français qui maîtrisent bien la langue, souvent, se destinent à d'autres carrières que l'enseignement.

Par ailleurs, les méthodes dont on se sert généralement pour l'enseignement du français langue étrangère (FLE) sont conçues pour être utilisées dans un maximum de pays et ne répondent pas toujours à la spécificité d'un enseignement bilingue. Ceci exige, de la part de l'équipe pédagogique, de remédier à ces manques et d'élaborer des documents et des supports propres. L'enseignement de la culture française est parfois rendu difficile à cause du manque de documents authentiques récents ou actualisés d'une part, d'autre part à cause du manque d'expérience ou de connaissance des professeurs turcs de français.

37 Au niveau de l'enseignement bilingue les méthodes de travail, les techniques d'accès au savoir, de construction de sens sont très différentes voire opposées entre le système scolaire français et turc. Cette difficulté nécessite de la part de l'équipe pédagogique une concertation régulière en vue d'une harmonisation méthodologique.

Enfin, apprendre le français, dans un milieu qui est loin d'être défini comme francophone est difficile : pour permettre à la langue française de devenir langue de communication et d'action, il est nécessaire de dynamiser un enseignement scolaire par des activités périscolaires et des animations en français (club théâtre, club journal, manifestations culturelles, etc.).

Mais dans la plupart des cas, le rapport de l'élève avec la langue seconde reste souvent scolaire. L'établissement scolaire est en général le seul référent quant à la langue et à la culture étrangères. Peu d'enfants ont l'occasion durant leur scolarité bilingue de profiter d'un séjour en France ou dans un pays francophone.

Si on fait une étude sur le parcours scolaire des personnes promises à un avenir remarquable dans le pays, on peut facilement repérer que la majorité vient des établissements secondaires bilingues.

Toutefois, suivre une scolarité bilingue est un projet qui n'est pas toujours facile dans un environnement monolingue. Cela exige beaucoup d'effort et de motivation de la part des élèves. C'est aux partenaires de cet acte éducatif, à savoir les élèves, les responsables pédagogiques, les familles, de maintenir une volonté et un travail permanents pour permettre à l'enfant de devenir bilingue et biculturel. 
INDEX

Index géographique : Turquie

Mots-clés : enseignement bilingue, langue anglaise, langue allemande, langue française, apprentissage d'une langue étrangère, section bilingue, apprentissage du français

\section{AUTEURS}

\section{JOËLLE SCHWENDIMANN CASSEL}

Coordonnatrice pédagogique, lycée Tevfik Fikret, Ankara, Turquie

\section{ESER GERDANLI}

Chef du département de français, lycée Tevfik Fikret, Ankara, Turquie 